\title{
Supply Chain Simulation with Switching Adaptive Model Predictive Control Methodology
}

\author{
Chunling Liu ${ }^{1} 2^{\star}$, Jizi Li ${ }^{2}$,Junfeng Wang ${ }^{1}$ and Yangjie Tian ${ }^{1}$ \\ ${ }^{1}$ School of Mechanics Engineering and Automation, Wuhan Textile University, Wuhan, China, \\ ${ }^{2}$ Supply Chain System Research Center, Wuhan Textile University, Wuhan, China \\ chunringliu5@yahoo.com.cn
}

\begin{abstract}
Keywords: Model predictive control; Optimization method; Switching control; Across-chain coordination
\end{abstract}

\begin{abstract}
An adaptive multiple model predictive control (MMPC) method for an uncertain input-constrained neutrally stable supply chains system with control-relevant switching is presented. By employing an input-to-state stabilizing MPC as the multi-controller, switching adaptive MMPC is proposed for the system. Unlike previous methods for handling uncertainties on the basis of minimal MPC laws or techniques for linear parameter varying systems, the proposed MPC scheme employs model switching to deal with modeling uncertainties through adaptation; a best model is selected for the MPC law from time to time. The proposed scheme using finite prediction horizon guarantees global stability. Simulation results are given to show the effectiveness of the scheme.
\end{abstract}

\section{Introduction}

In recent years, the supply chain network structure is ever-changing, Firms are obliged to maintain high customer service levels while at the same time they are forced to reduce cost and maintain profit margins. Traditionally, firms can operate and coordinate their marketing, distribution, planning, manufacturing, and purchasing along their own single supply chain. But, in real business world, there are likely to be a couple of related single supply chains nearby. Not only these single supply chains compete with one another, but cooperation happens among them. Where does it occur? With the further development of industrial and specialization division, industrial cluster provide an environment to makeup multi-chains and promote their member cooperation between them in order to implement legality strategy for sharpening their edge of competitive advantages. Moreover, uncertain market demand also forces firms to adopt coordination policy from firm-wide cooperation to chain-wide cooperation, and even to a cross-chain cooperation so that firms can survive and thrive. On this basis, we refer to multiple of single supply chains located in industrial cluster as cluster supply chains. Researches regarding inventory management of supply chain mainly focused on a single supply chain, which only take into account inventory management and bullwhip effect at two or more than two tiers and ignore inventory transshipment from the other adjacent related single supply chain. Although some literatures refer to their studies in the context of supply chain networks, which looks like multi-chains, this 'networks' basically contain just one focal firm who is dominant in whole supply chain and superior to other upstream and downstream firms, due to fan-shaped structure formed by subordinating, this supply chain calls as chain networks firms (Chopra \& Meindl, 2004), but the one focal firm, rather more than one, plays a leadership role in making decision regarding as facility location, production capacity, inventory policy, transportation mode and batch size etc, in this way, this kind of chain network is indeed a single supply chain rather than multi-chains system.

Model predictive control (MPC) is a receding horizon strategy, where the control is computed via an optimization procedure at every sampling instant. It is therefore possible to handle physical constraints on the input and/or state variables through the optimization. Over the last decade, there have been many stability and robustness results on constrained MPC. Because of this, MPC is recognized as one of the most successful control schemes in both theory and practice.

Perea-Ló PEZ et al. (2003) proposed a SCM model based on the reasonable hypothesis from the 
raw materials suppliers to the consumers, and parameters of the various nodes were analyzed by the policy of overall optimization target. Box et al. (1978) proposed a time series forecasting method in details, and under a series of white noise in demand, an autoregressive moving average model ( ARIMA ) was employed, which is based on the past experience obtained. In this paper, a switching adaptive MPC for input-constrained neurally stable linear plant is presented. As the controller in the proposed switching adaptive control scheme, the input-to-state stabilising MPC is employed. As seen later, employing such a robust MPC against the estimation error is the key to design the adaptive MPC. For the switching logic, the control-relevant switching logic recently devised is employed.

\section{MPC Model for a Class of Supply Chains System}

In recent years numerous articles in supply chain modeling have addressed the issue of inventory coordination. Goyal and Gupta (1989) classified the literature dealing with the integrated models into four main classes. And Houqe and Goyal (2000) extended the idea of producing a single product in a multi-stage serial production system with equal and unequal sized batch shipments between stages. Khouja (2003) considered the case a three-stage non-serial supply chain and developed the model to deal with three inventory coordination mechanisms between the chain members. Cárdenas-Barrón (2007) formulated and solved an n-stage-multi-customer supply chain inventory model where there is a company that can supply products to several customers. Chung and Wee (2007) considered an integrated three-stage inventory system with backorders. They formulated the problem to derive the replenishment policies with four-decision-variables algebraically.

As mentioned earlier numerous articles in inventory modeling have been written under a single supply chain. Therefore, there is a need to analyze models that relax the usual assumptions to allow for multiple supply chains network inventory coordination with the across-chain transshipment.

Suppose the supply chain upstream node always satisfy the demand from downstream node. This paper considers two models: inventory model and demand model. Inventory model is used to maintain inventory balance of supply chain knods, demand model used to forecast future demand.

System parameters are stated as follows:

$I(t)$ Product inventory of a node at time $\mathrm{t}$

$M_{i n}{ }^{(t)}$ Product quantity from the upstream node

$M_{\text {out }}(t)$ Product quantity to a downstream node upstream node

$O_{\text {out }}$ Order quantity of the downstream node $\quad L$ Delay time of arrival order

$P_{i n}$ The purchase price of the product from the upstream node $P_{\text {out }}$ The price of the product to a downstream node

$P_{\text {stock }}$ Products inventory cost cost

$R(t)$ The total product yield

$C_{\text {pur }}$ Products procurement

$C_{\text {stock }}$ Inventory cost

Inventory Model. In order to simplify the analysis of supply chains, suppose there are three nodes in every supply chain: supplier, manufacturer, and retailer. Income is from the downstream nodes, and the cost contains purchasing cost and maintain inventory cost from an upstream node. Assume that each node proceeds from the difference between income and cost. Fig. 1 shows the structure diagram of two supply chains (where, across-chain mutual replenishment is included.). 


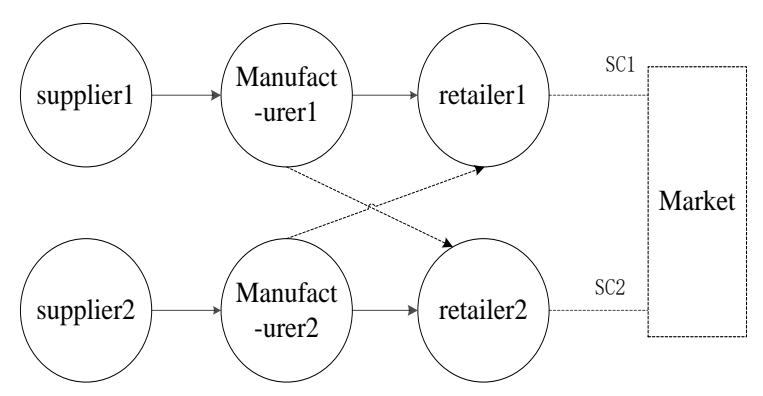

Figure 1. The two supply-chain structure with across-chain mutual replenishment

Therefore, from the above supposition and the supply chains structure, the inventory balancing can be expressed by the following equation:

$$
I(t+1)=I(t)+M_{\text {in }}(t)-M_{\text {out }}(t)
$$

It is assumed that the product volume from upstream nodes is always adequate, but demand is random, so relationship of a node and its upstream node may be formed by the following formula:

$$
M_{\text {in }}(t+L)=O_{\text {in }}(t)
$$

Here, $\mathrm{L}$ is delay time of the arrival order. Thus the relationship between a node and its downstream node is formed as:

$$
M_{\text {out }}(t)=\left\{\begin{array}{cl}
O_{\text {out }}(t), & I(t)+M_{\text {in }}(t) \geq O_{\text {out }}(t) \\
I(t)+M_{\text {in }}(t), & I(t)+M_{\text {in }}(t)<O_{\text {out }}(t)
\end{array}\right.
$$

The constraints can be seen as rigid ones. A node must meet the needs of downstream node as possible as it can.

Set $P_{\text {in }}$ represents purchase price from an upstream node, $P_{\text {out }}$ the product price to downstream node, and $P_{\text {stock }}$ node inventory cost. To simulate a real market operation process, we assume the sectional relation between the total monetory amount of orders and cost is as the following: (where, $C_{\text {pur }}$ represents procurement costs)

$$
C_{p u r}(t)=\left\{\begin{array}{cc}
P_{1} O_{i n}(t), & 0<O_{i n}(t) \leq O_{1}, \\
P_{1} O_{1}+P_{2}\left(O_{i n}(t)-O_{1}\right), & O_{1}<O_{i n}(t) \leq O_{2} \\
\vdots & \vdots \\
\sum_{i=1}^{n-1} P_{i} O_{i}+P_{n}\left(O_{i n}(t)-O_{n-1}\right), & O_{n-1}<O_{i n}(t) \leq O_{n}
\end{array}\right.
$$

Here, $P_{i}$ is the different purchase price (the actual value of $P_{i n}$ ) according to the order quantity; $O_{i}$ is the boundary constraint of order volume. Set $R(t)$ represents the product of product price $P_{\text {out }}$ and product orders $O_{\text {out }}(t)$, namely as the total product yield, which is refered by the following equation: ( $P_{\text {out }}$ is a constant)

$$
R(t)=P_{\text {out }} O_{\text {out }}(t)
$$

Assume $C_{\text {stock }}$ and stocks shows a linear relationship, there is

$$
C_{\text {stock }}(t)=P_{\text {stock }} I(t)
$$

Therefore, for a node, its total economic benefits are as follows:

$$
J_{\text {profit }}(t)=R(t)-C_{\text {pur }}(t)-C_{\text {stock }}(t)
$$

In the supply chains, a node's ultimate goal is to achieve the maximum profit, but also to meet the demand in the downstream node. Here, $O_{i n}(t)$ is the manipulated variable, $O_{\text {out }}(t)$ can be viewed as a random perturbation. In order to realize this purpose, it is necessary to forecast demand model of $O_{\text {out }}(t)$.

Demand Model. Box et al. in 1978 first proposed a time series prediction model: ARIMA model. 
Erdogdu (2007) used ARIMA model to predict Turkey's national electricity demand, the results showed that the model was an effective forecast model. Niu and Qian (2007) applied ARIMA model to a kind of semiconductor demand forecasting. Here, demand model may be choosen as:

$$
A\left(z^{-1}\right) y(k)=B\left(z^{-1}\right) u(k-1)+C\left(z^{-1}\right) \xi(k) / \Delta
$$

Here, $A\left(z^{-1}\right) 、 B\left(z^{-1}\right)$ and $C\left(z^{-1}\right)$ respectively represent $\mathrm{N}, \mathrm{m}$ and n-order $z^{-1}$ polynomial, $\Delta=1-z^{-1} ; y(k) 、 u(k)$ and $\xi(k)$ respectively represent output, input and the white noise sequence of 0 mean. Based on this method, we could design a model predictive controller through feedback correction to forecast future demand.

\section{Model Predictive Control Strategy}

Model predictive control (MPC) is a kind of control algorithm that can solve the discrete-time optimal control problems. It maximize the system economic objective function by means of an optimal control law (Camacho and Bordons, 1995). MPC control algorithms are considered as one of advanced optimal control techniques for industrial process control. And it has a significant impact, because the MPC control technology can effectively resolve routine constraints on the equipment and safety (Maciejowski, 2002). MPC may generally be divided into the following three parts: the predictive model, rolling optimization and feedback correction. In process control, it is the target to maintain a stable process.

Model Predictive Control Objective. Through the above analysis, the goal of the system model can be expressed as follows:

$$
\begin{aligned}
& J_{\text {profit }}=\sum_{i=1}^{P} R(t+i \mid t)-\sum_{i=1}^{P} C_{\text {pur }}(t+i \mid t)-\sum_{i=1}^{P} C_{\text {stock }}(t+i \mid t) \\
& \text { where, } \quad R(t+i \mid t)=P_{\text {out }} \hat{O}_{\text {out }}(t+i \mid t), \quad C_{\text {stock }}(t+i \mid t)=P_{\text {stock }} I(t+i \mid t), \quad i=1,2, \ldots, P, \\
& C_{\text {pur }}(t+i \mid t)=\left\{\begin{array}{cc}
P_{1} \hat{O}_{\text {in }}(t+i \mid t), & 0<\hat{O}_{\text {in }}(t+i \mid t) \leq O_{1}, \\
P_{1} O_{1}+P_{2}\left(\hat{O}_{\text {in }}(t+i \mid t)-\hat{O}_{1}\right), & O_{1}<\hat{O}_{\text {in }}(t+i \mid t) \leq O_{2}, \\
\vdots & \vdots \\
\sum_{i=1}^{n-1} P_{i} O_{i}+P_{n}\left(\hat{O}_{\text {in }}(t+i \mid t)-\hat{O}_{n-1}\right), & O_{n-1}<\hat{O}_{\text {in }}(t+i \mid t)
\end{array}\right.
\end{aligned}
$$

In order to avoid demand mutations, we assume that the inventory model is under soft constraints. At the same time set the maximum and minimum constraints of predicted inventory amount as follows:

$$
I_{\min } \leq I(t+i \mid t) \leq I_{\max }, i=1,2, \ldots, P
$$

Because $O_{\text {out }}$ is random, $O_{\text {in }}$ fluctuates accordingly. Given $C_{p u r}$ is sectionally linear(influenced by $O_{i n}$ ), the objective function is not a standard linear programming problem. So the control procision will be interfered if a unified model is applied to the system. Therefore, we divide the system into several linear subsystem and use multiple models switching method to solve this problem.

\section{Multiple Models Switching Strategy}

Model switching based on multiple model control is a new control mode used to deal with model uncertainty. Multiple models switching mechanism is introduced into predictive control, which constitutes multiple-model switching predictive-control algorithm. The current main researches are: (1) controller switching strategy based on predictor; (2) a controller switching strategy for predetermined path, in which controller switching time relates to the controlled object variables. In this paper by the set switching law in advance, the predictive control switcher completes multiple models switching. Fig. 2 shows the structure diagram for multiple models switching system. 


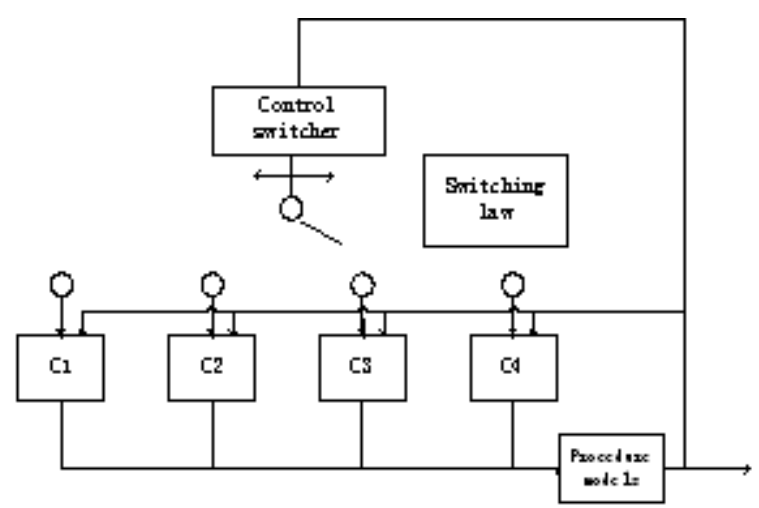

Figure 2. Schematic diagram of multiple models

In the graph, C1, C2, C3 and C4 express 4 system models in supply chain. With the market demand changing, the model predictive controller switches to the appropriate model according to the given switching law, for achieving the maximum economic benefits while the needs of the whole supply chains are met.

In any control strategy, the question of closed-loop stability is of great importance. The general approach in stability analysis of MPC is that the performance index is interpreted as a Lyapunov function and shown to be monotonically decreasing. Various predictive control strategies with stability guarantee have been proposed. A detailed review can be found in literatures[Allgower, \& Mayne]. These approaches either impose contractive constraints on the system states or drive the system states to zero or inside a region where the control law is obtained from a stabilizing linear controller. The stability results of these formulations depend on the feasibility of the control law. This issue has recently been addressed by [El-Farra,2004]. In their work, a controller strategy that combines the bounded control approach with MPC for constrained linear systems was proposed. The main idea is the utilization of bounded control whenever MPC results in infeasibility and hence guarantee that the system will evolve in the stability region defined by the bounded controller.

Closed-loop stability in multiple model/control approaches has also been studied [Gundala, Narendra \& Tian] since designing local controllers that stabilize each individual model may not result in a stable global closed-loop system. In general, the use of multiple models in a control structure necessitates a means of switching among the available models to the one that best describes the current operating condition. The switching from one model/controller to another based on a logical argument (supervisory scheme) results in a hybrid system. An approach that has found wide utility in stability analysis of hybrid systems is multiple Lyapunov functions [Branicky,1994, 1998]. The idea originates from the fact that it may not be possible to find a common Lyapunov function that ensures stability for all the subsystems and the global system. A closely related work is the stability analysis of piecewise linear systems by [Rantzer, 1998] in which piecewise quadratic Lyapunov functions were constructed using convex optimization in terms of linear matrix inequalities (LMIs) as an alternative to a globally quadratic Lyapunov function.

With regard to the multi-model switching control, Liu G. Q. analyzed and proved the feasibility and stability of the system, and showed practicability and stability of the proposed switching control algorithm combined with simulation example. Here thus simula discussion will not be developed, but the actual case analysis with two chains will be made to illustrate that the supply chain can achieve the goal of optimal performance under this control strategy.

\section{Simulation Research}

Suppose the supply chain is a multiple-input single-output system. Here the methods of order-up to level ( OUL ) and model predictive control were compared. The OUL method can use the following expression: 


$$
O_{i n}(t)=I_{s p}-I(t)
$$

Wherein, $I_{s p}$ expressed inventory setting value, $I(t)$ in-time inventory. The actual demand is supposed to produce from the formula:

$$
O_{\text {out }}(t)=\frac{1+0.5 z^{-1}}{1-0.4 z^{-1}} \xi(t)+100+\zeta
$$

$\zeta$ is the random number between -0.1 and 0.1 (time unit, day). This means that the fluctuations in demand were 100 or so. Purchase cost was composed of the following formula:

$$
C_{\text {pur }}(t)=\left\{\begin{array}{cc}
10 O_{i n}(t), & 0<O_{i n}(t) \leq 100, \\
1000+8\left(O_{\text {in }}(t)-100\right), & O_{\text {in }}(t)>100
\end{array}\right.
$$

Set $P_{\text {stock }}=0.5$. In order to compare with OUL method, we assumed that the two methods had the same average inventory

$$
I(t)+O_{\text {in }}(t-1)-O_{\text {out }}(t)=\left(I_{\max }+I_{\min }\right) / 2
$$

At the same time hypothesis the expected value of $I(t)$ and $I(t-1)$ is the same, and the expected value of $O_{\text {out }}(t)$ was 100 . Thus there was

$$
I_{s p}=\left(I_{\max }+I_{\min }\right) / 2+100
$$

Here time series simulation in demand for 30 days was traced. We compared the two methods of MMPC and OUL in different prediction horizon. As shown in Table 1, the largest inventory $I_{\max }$ $=300, . \quad I_{\min }=250$ when $P_{\text {out }}$ is 10

Table 1 Inventory under MPC method and OUL method

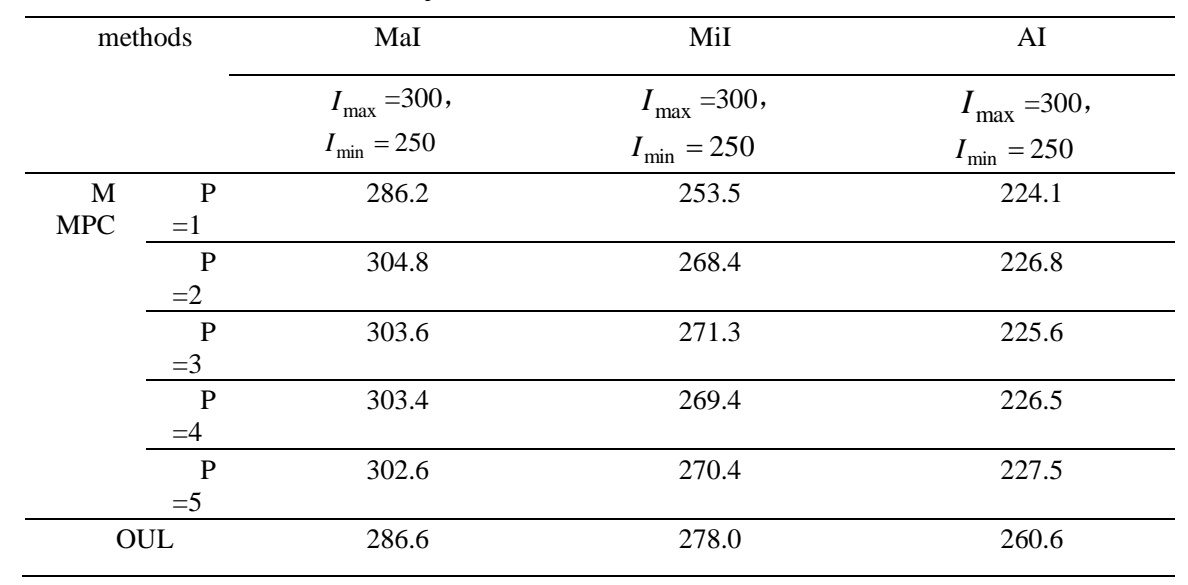

(MaI: represents the largest inventory; MiI: the minimum inventory; AI: the average inventory. MMPC: multiple model predictive control; OUL: order-up to level )

Because purchase price of the system is sectionally linear, fluctuational stock can use this property to get more profit. Table 1 shows that the inventory stability by OUL method is better than MMPC method, but the MPC method can effectively control the bullwhip effect in supply chain system in a period of time, In addition, the MMPC method can get more economic benefits than OUL method. And because the purchase price is a sectionally linear function, loose constraints also can obtain more profit. $P_{\text {out }}$ Similarly has important implications on the economic efficiency, as $P_{\text {out }}$ quite high, economic benefits fluctuations under the MMPC method at different time domain is less than that of OUL method (shown in Fig. 3).

As you can see, when $P_{\text {out }}=10, I_{\max }=300, I_{\min }=250$, the maximum profit $(\mathrm{P}=2)$ by the MMPC method is higher by $6.67 \%$ than the OUL method. Both the two methods can greatly improve the economic benefit. However, when $P_{\text {out }}$ is relatively low, the different profit difference of two methods will be expanded(shown as Fig. 3) 


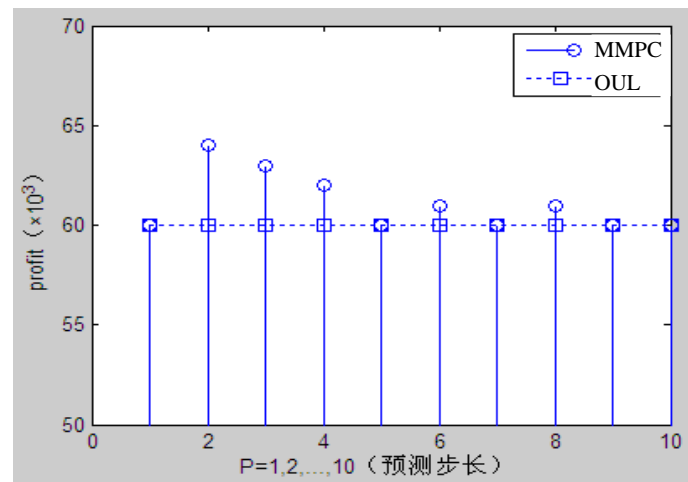

Figure 3. Profit comparison of MMPC method and OUL method $\left(P_{\text {out }}=10\right)$

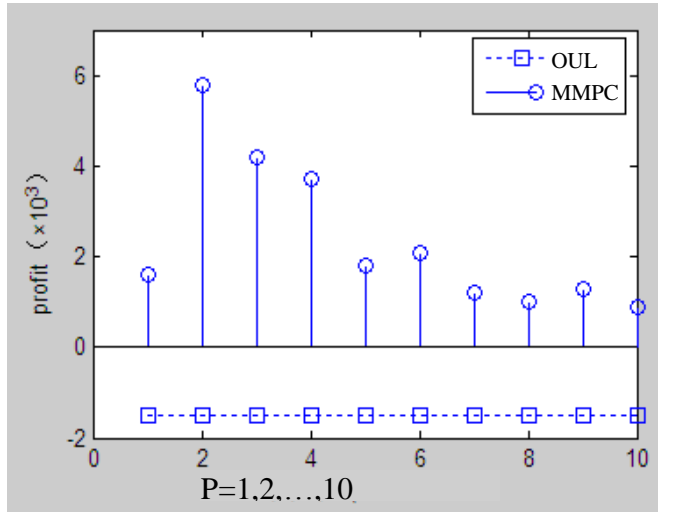

Figure 4. Profit comparison of MMPC method and OUL method

$$
P_{\text {out }}=5
$$

As shown in Fig. 4, when $P_{\text {out }}=5 I_{\max }=300, I_{\min }=250$ using the MMPC method still could obtain more stable profit, while the OUL method obtained negative return at the time. This simulation study also shows that the system with the shorter forecasting time domain could achieve more earnings than that with longer time domain. As the market competition becomes more intense, $P_{\text {out }}$ will become more and more low, therefore, the selection of an effective and economic order decision has become more and more important.

\section{Acknowledgment}

The paper is supported in part by China NASFC (National Science Function Committee) (71472143), This research is also supported by China Education Ministry (15YJA630035).

\section{References}

[1] J.M. Maciejowski. Predictive Control with Constraints. Person Education Limited, Edinburgh Gate, UK., 2002

[2] M. Kothare, V. Balakrishnan, and M. Morari. Robust Constrained Model Predictive Control using Linear Matrix Inequalities, Automatica, 32(1996), 1361-1379.

[3] J. Gao, R. Ratwardhan, K. Akamatsu, Y. Hashimoto,G. Emoto, S. Shah, and B. Huang. Performance Evaluation of Two Industrial MPC Controllers, Control Engineering Practice, 11(2003), 1371-1387.

[4] P. Fleming, and A. Pashkevich. Application of Multi-objective Optimisation to Compensator Design for SISO Control Systems, Electronics Letters, 22 (1986), 258-259.

[5] H. Min, G. Zhou. Supply chain modeling: past, present and future. Comput. Ind. Eng., 2002, 43(1-2): 231-249.

[6] P. Lin, D.S. Wong, S. Jang, S. Shieh, J.Z. Chu. Controller design and reduction of bullwhip for a model supply chain system using z-transform analysis. J. Process Control, 2004, 14(5):487-499.

[7] E. Perea-López, B.E. Ydstie, I.E. Grossmann. A model predictive control strategy for supply chain optimization. Comput. Chem. Eng., 2003, 27(8-9): 1201-1218.

[8] G.E.P. Box, G.M. Jenkins, G.C. Reinsel. Time Series Analysis: Forecasting and Control. John Wiley, San Francisco, USA, 1978. 
[9] M. Ohshima, H. Ohno, L. Hashimoto, M. Sasajima, M. Maejima, K. Tsuto, T. Ogawa. Model predictive control with adaptive disturbance prediction and its application to fatty acid distillation column control. J.Process Control, 1995, 5(1):41-48.

[10]D.F. Pyke, \& M.A. Cohen. Multiproduct integrated production distribution system. European Journal of Operations Research, 74(1994), 18.

[11]A.G. Lagodimos. Multi-echelon systems: a service measure perspective. European Journal of Operations Research, 95(1996), 241. 